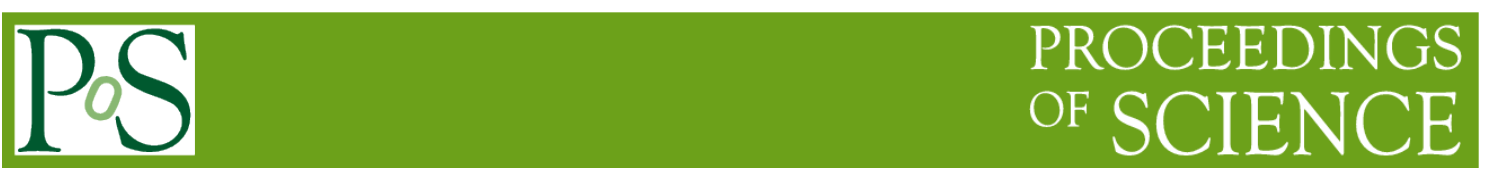

\title{
Research on Mobile Robot SLAM Based on Laser Range Finder
}

Ri Gao'

Beijing University of Civil Engneering And Architecture

BeiJing, 100044,China

E-mail: 13691416707@163.com

\section{Lei Zhang}

Beijing University of Civil Engneering And Architecture

BeiJing, 100044,China

E-mail: $188109726060163 . \mathrm{com}$

\section{ShuJing Zhang}

Beijing University of Civil Engneering And Architecture BeiJing, 100044,China

E-mail: $136914167070163 . \mathrm{com}$

[Purpose] Robot positioning and construction of the environmental map is an important guarantee for its own task. In the unknown environment, problems of robot positioning and map creation are interdependent. Researchers has put forward the problems of simultaneous robot positioning and map creation (SLAM). [Method] In this paper, the landmark extraction algorithm based on RANSAC method is studied for the twodimensional environment, and the algorithm is improved according to the application requirements of SLAM problem. Finally, the mobile robot is used to experiment on the landmark extraction and EKF-SLAM algorithm. [Results] The results show that the algorithm of Landmark extraction and EKF-SLAM is valid.

CENet2017

22-23 July 2017

Shanghai, China

${ }^{1}$ Correspongding Author 


\section{Introduction}

The autonomous navigation of a mobile robot means that the robot moves autonomously from one place to another, effectively exploring the unknown area and completing the given task. This requires the robot in the unknown area to determine their own position in the environment, which is the robot self-positioning problem. And the positioning of the problem and the creation of the environment map is a complementary and continuous iterative process. Therefore, the robot can identify and analyze the external environment in the unknown environment where its position is uncertain, and then creates the map, locates and navigate $s$ with it. That is the simultaneous positioning and map creation (SLAM) of the mobile robot.[1]

At present, the research on SLAM mainly focuses on improving the real-time, data association and loop closure. Some researches are also performed on the environmental representation, map creation and location navigation to identify the problem of autonomous navigation SLAM in unknown environment with the mobile robot. This paper discusses the design of a scheme for constructing the 2D visualization landmarks. [2] The SLAM based on the integration of sonar information and visual information is studied to improve the accuracy and robustness of the robot SLAM.[3] In the indoor environment, Instrument and monocular vision SLAM are applied to achieve the mobile robot's autonomous navigation task.[4]

However, in the unknown complex environment, there are both irregular static obstacles and dynamic obstacles in the space, which makes the sensor data have a high degree of uncertainty. Therefore, this paper studies the landmark extraction based on the RANSAC method for the two-dimensional environment with the line landmarks Algorithm, and according to the SLAM, problem application requirements of the algorithm have been improved.

\section{The Mobile Robot System Model Established}

\subsection{Coordination System}

The relationship between the environmental referential coordination system and the robot coordination system is shown in the following Figure 1.

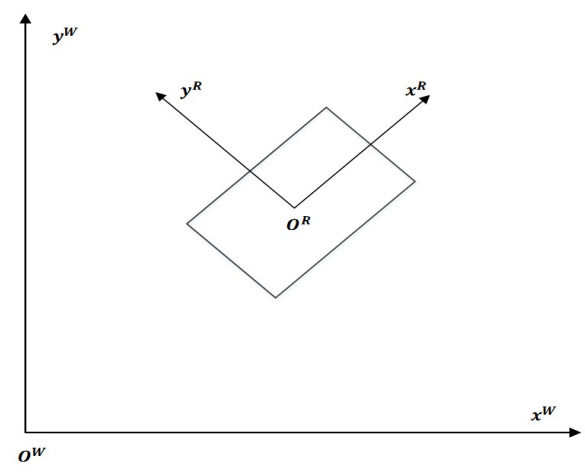

Figure 1:Mobile Robot Coordination System

The origin of the robot coordination system is located at the center of the rotation of the robot. The Rx axis is pointing in front of the robot and Ry is pointing to the left side of the robot. The environment referential coordination system remains the same in the robot operating 
environment. The SLAM problem studied for the unknown environment usually initializes the environmental referential coordinattion system with the initial robot coordinattion system.

\subsection{Environmental Model}

In this paper, the general indoor environment is selected as the object and in the twodimensional space SLAM problem study, the plane is chosen in the line as a landmark. [5]Save all the landmarks in the environment as a landmark library and the map is achieved, as shown in the following figure.

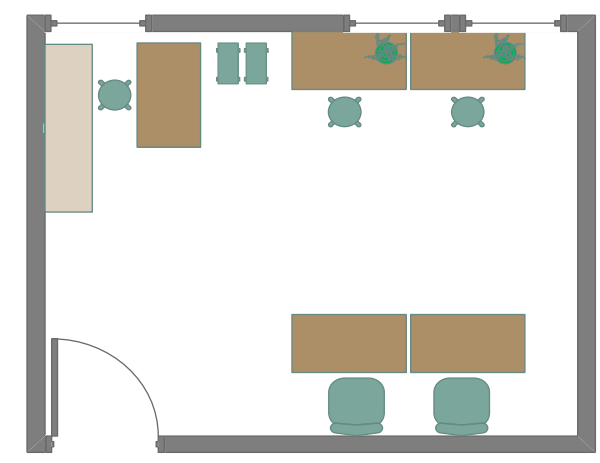

Figure 2: Indoor Environment

Taking the unknown environment into account where only the coordinates of the origin are determined, we choose the environment referential coordination system with origin line on the projection as a landmark, as shown in Figure 3.

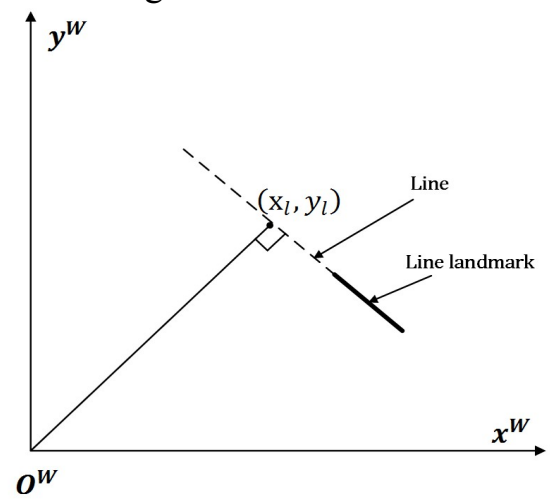

Figure 3: Select A Placemark From the Line on Which the Line is Located

The two-dimensional position vector in the figure can be used to filter the calculation, but can't be applied to fully describe the position of the line segment in the environment. To reach this aim, the establishment of landmarks is used to preserve the two ends of the line in the polar coordination system under the angular coordinates, as shown in Figure 4. 


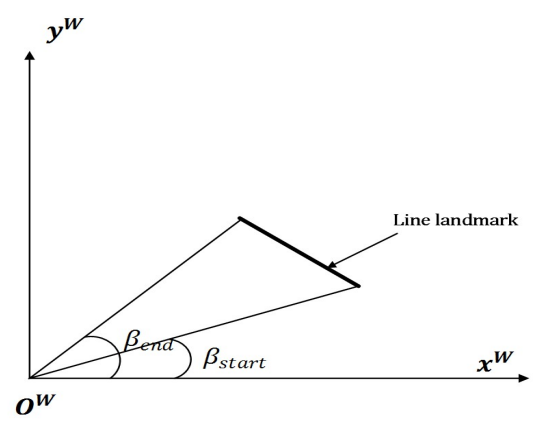

Figure 4: The Angular Coordinates of the Line Endpoint

\subsection{Sensor}

In this paper, the HOKUYO UST-10LX model of the laser range finder with scanning area $270^{\circ}$, the measurement range $0.06 \mathrm{~m}-10 \mathrm{~m}$ and distance and angle resolution $\pm 40 \mathrm{~mm}$ and 0.25 ${ }^{\circ}$ (relative scan data points 1081) is adopted which can meet the needs of robot in this paper.

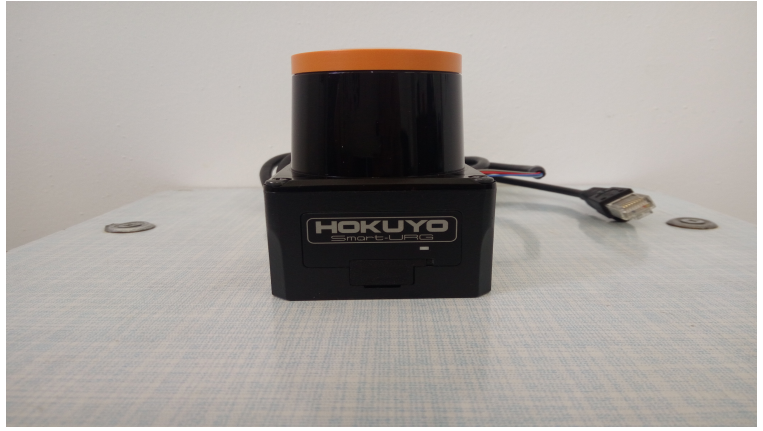

Figure 5: Laser Range Finder

The measurement output of the laser range finder is the polar coordinate of the object:

$$
Z i=(\rho i+W \rho, \alpha i+W \alpha) T i=1, \ldots, N
$$

Where $W \rho$ and $W \alpha$ are the measurement errors of the distance and direction respectively.

\section{Study on the EKF-SLAM Problem of Line Segment Landmark Environment}

In this section, the RANSAC parameter estimation method is used to extract the landmarks, and the RANSAC algorithm is improved according to the application of EKFSLAM.

\subsection{Draw Line Landmarks}

In order to facilitate the data association process and describe the line landmark in the map, we need to record the two endpoints of the line segment while extracting the landmark, and select the observation direction angle of the end of the line to describe the endpoint position as shown in Figure 6. 


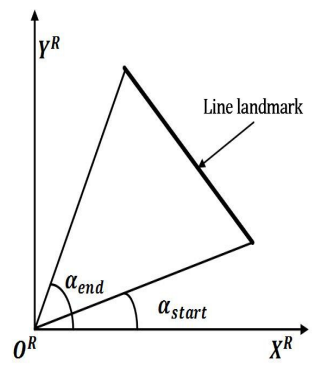

Fgure 6: Take A line of Landmarks

\subsection{Landmark to Weight}

The observation of the landmarks in the environment at each moment of the robot should be unique, that is, the robot cannot observe the same landmark twice. To avoid duplication of the same landmarks, the samples in the consistent set are removed from all data sample. Let's set $\mathrm{D}$ each time when the landmarks are successfully extracted.[5]

\subsection{Improve the RANSAC Algorithm Flow}

The improved RANSAC algorithm applied to the line segment extraction is shown in the following figure.

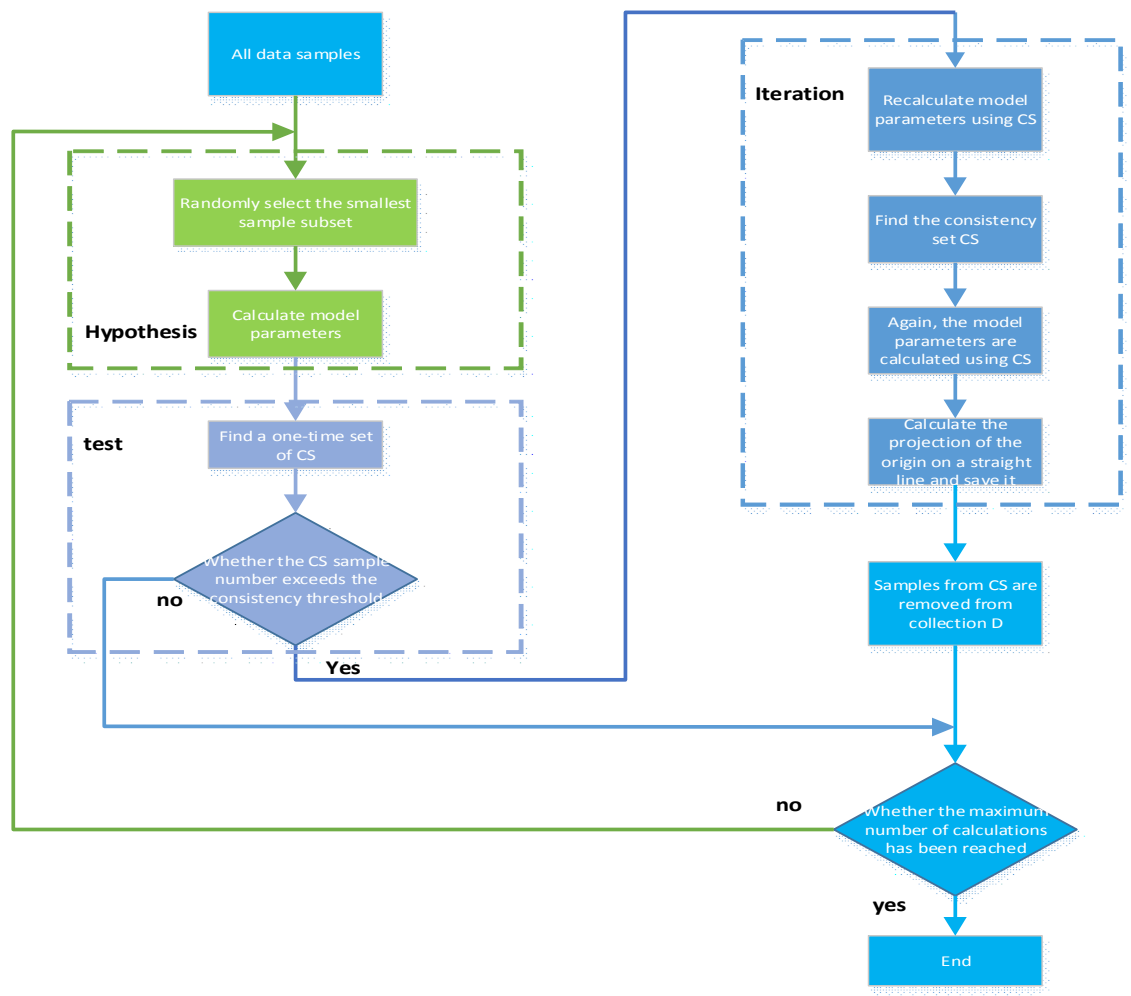

Figure 7: EKF-SLAM Improved RANSAC Algorithm Flow

\section{EKF-SLAM Experiment on Segment Landmark Environment}


The SLAM experiments are performed in an indoor environment as shown below by using the Opti-Track visual positioning system to measure the position and heading of the robot. There are five landmarks in the environment, and the map of the landmark location is shown in the figures below .

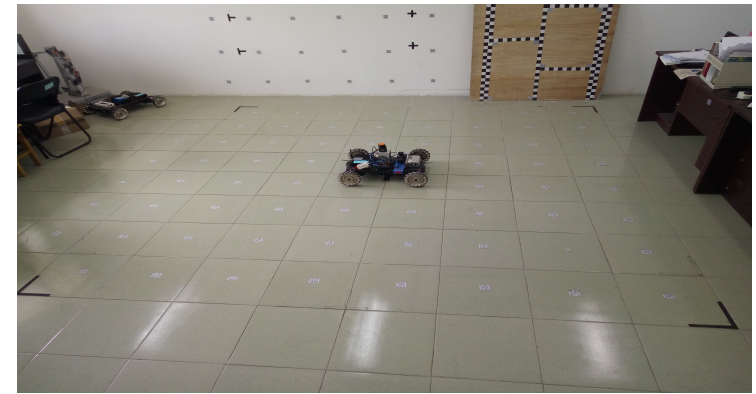

Figure 8: Part of the Experimental Environment

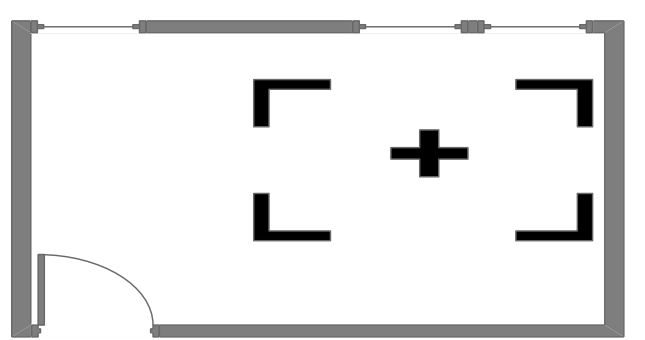

Figure9: Experimental Environment Plan

Robot with the "priority straight to the road, turn left" principle in the environmental independent obstacle movement when running the EKF-SLAM algorithm. During the experiment, the positioning and map creation results and the robot running status are shown in the following Figure10 and11.

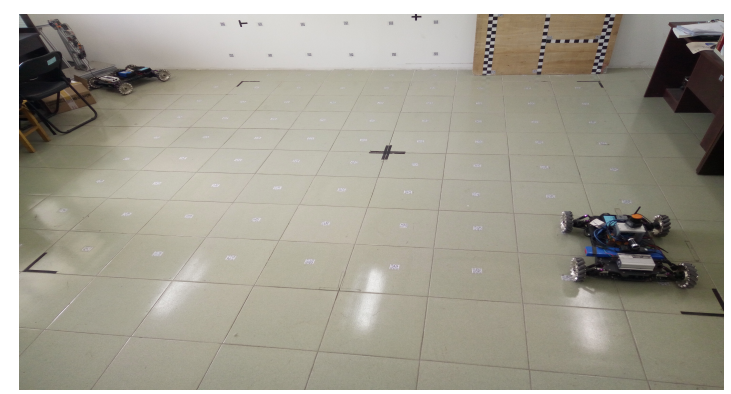

Figure 10: Robot Status

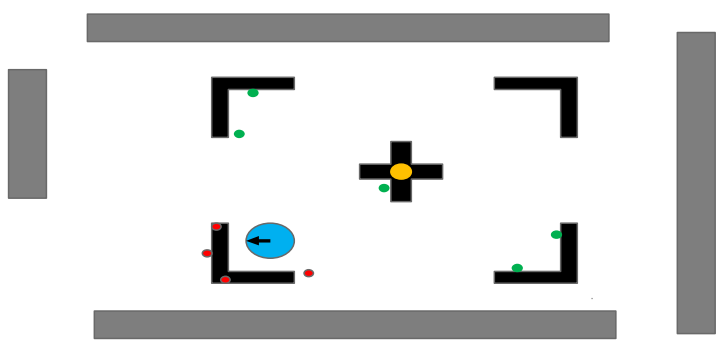

Figure 11: Robot Pose and Map

In the figures, the blue dots and the black short lines on the dots indicate the estimated pose of the robot, and the black line is the robots' gradually build landmarks. The orange dots indicate the origin of the environment referential coordination system, and the green dots indicate the projection of the origins of the environmental referential coordination system on each line segment. The red dots mean that the robot is pumping from the environment in real time. The results of robot positioning and map creation are shown as below. 


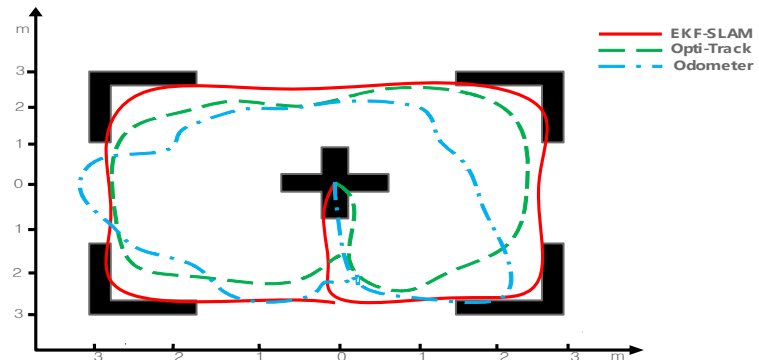

Figure 12: Experimental Results

In the three curves, the red solid line indicates the EKF-SLAM. The algorithm is used to estimate the motion trajectory of the robot. The green dotted line indicates the robot motion trajectory measured by the Opti-Track visual positioning system. The blue dotted line indicates the robot trajectory of the odometer direct output. As revealed from the figure, the EKF-SLAM algorithm obtains the robot position estimation accurately.

\section{Conclusion}

In this paper, a complete solution to the SLAM problem, including landmark extraction, Kalman filter and data association, is proposed in the two-dimensional environment described by line landmark. The indoor SLAM experiment system of indoor environment is established on the mature and reliable robot platform with the visual positioning system. The effectiveness of the EKF-SLAM algorithm is verified by this system.

\section{References}

[1] Huang Mingden, Xiao Xiaoming, Cai Zixing and so on. Research on Extraction Method of Robot Local Environment[J]. Computer Measurement and Control, 2007,15 (2): 241-244

[2] Luo Ronghua, Hong Bingrong Research on simultaneous location and map creation based on information fusion [J]. Journal of Harbin Institute of Technology, 2004, 36 (5): 566-569.

[3] Smith R, Self M, Cheeseman P. Estimating ncertain spatial relationships in robotics [J]. IEEE Robotics and Automation, 1987, 4:850.

[4] Ya Zhuang, Wang Wei, Wang Ke and so on. Mobile robot based on laser ranging and monocular vision of indoor simultaneous positioning and map building [J]. Journal of Automation, 2005, 31 (6): 925-933.

[5] Sebastian Thrun. Probabilistic Algorithms in Robotics [J]. AI Magazine, 2000, 21(4): 93-109. 